\title{
Assessment of Different Expression Strategies for the Production of a Recombinant Lipoprotein Vaccine in Plants
}

\author{
Anna Hennig ${ }^{1,2}$, Yvonne Reinders ${ }^{2}$, Anatoli Giritch ${ }^{3}$, Jörg Reinders ${ }^{2}$ and Heribert Warzecha ${ }^{1,2, *}$
}

${ }^{I}$ Darmstadt University of Technology, Institute of Botany, Darmstadt, Germany; ${ }^{2}$ University of Wuerzburg, Department of Pharmaceutical Biology, Julius-von-Sachs-Institut für Biowissenschaften, Wuerzburg, Germany and Icon Genetics GmbH, Biozentrum Halle, Halle/Saale, Germany

\begin{abstract}
The ability of plants to serve as a production system for bacterial lipoprotein vaccines has been investigated. First, the effect of high-level expression of the Borrelia burgdorferi outer membrane protein A (OspA), a prototype vaccine against Lyme disease, has been examined by a proteomics approach. Analysis by 2D-PAGE of wild type tobacco plants and transplastomic plants accumulating recombinant OspA showed no apparent differences in protein pattern except for OspA. However, presence of the bacterial signal sequence limits transgene accumulation. As an alternative approach OspA was produced in Nicotiana benthamiana plants by transient expression via a deconstructed tobacco mosaic virus-based system. While rapid expression of OspA could be achieved, no palmitoylation occurred with the genuine bacterial sequence. In contrast, modification of the N-terminus with an eukaryotic sequence motif resulted in palmitoylation of OspA. This study shows that plants provide multiple expression strategies and could serve as a versatile production platform for recombinant lipidated subunit vaccines.
\end{abstract}

Key Words: Plant vaccine, lipoprotein, protein palmitoylation, outer surface protein A, chloroplast transformation.

\section{INTRODUCTION}

Bacteria exhibit on their outer surface a diverse set of proteins sharing a unique lipid modification. The so called bacterial lipoproteins play important roles in numerous physiological processes $[1,2]$ and also in host-pathogen interaction and pathogenesis. They are important targets for the immune system and for several pathogens, lipoproteins have been identified as potential vaccine candidates, e.g. from Streptococcus sp. [3] or Neisseria meningitides [4]. The most prominent example is the outer surface protein A (OspA) of the Lyme disease agent Borrelia burgdorferi, which has been already used as a vaccine in humans $[5,6]$. In the case of OspA it has been shown that the unique lipid moiety is essential for immunogenicity and that a non-lipidated form did not induce protective immunity in mice $[7,8]$. Therefore, production of the unique lipoproteins so far relies on bacterial expression systems.

Bacterial lipoproteins contain a typical N-terminal signal peptide recognized by the enzyme diacylglyceryl transferase (Lgt), which attaches a diacylglycerol to the thiol of a conserved cysteine which is an invariant part of the lipobox motif [9]. Cleavage of the signal peptide by signal peptidase II results in the unique Pam2Cys-structure at the amino terminus of the protein. Contingently, a third palmitic acid could be attached to the free amino group in Gram- bacteria, resulting in a Pam3Cys-structure, as present in OspA. It has been shown that OspA does not accumulate to very high levels when expressed in E. coli possibly due to interference with the export of host proteins [10]. Hence, production of large amounts of lipoproteins is limited by the processing capacity

*Address correspondence to this author at the Darmstadt University of Technology, Institute of Botany, Schnittspahnstr. 3-5, 64287 Darmstadt, Germany; E-mail: warzecha@bio.tu-darmstadt.de of the host bacterium. As an alternative system, we used plants as an expression host for OspA.

Plants have been widely assayed as a production platform for recombinant proteins and in particular for subunit vaccines $[11,12]$. Especially the use of chloroplast transformation has enabled the accumulation of recombinant proteins at extraordinary high levels. In the case of the TetC fragment from Tetanus toxin, $25 \%$ of the total cellular protein (TSP) could be achieved by expression in the chloroplast [13], and $22 \%$ TSP of a cholera toxin fusion could be produced [14]. Those are two examples of many showing that transplastomic plants have an enormous potential as protein factories. However, generation of plants is time consuming and frequently transgene expression reduces viability of plants [13, 15].

As an alternative, viral vectors can be used for transient expression in plants when the gene of interest is placed under the control of the strong coat protein promoter. The advantage of the system is, that extremely high levels of recombinant protein can be produced due to viral amplification in a very short time (days) [16]. Especially the system using in planta assembly of complete viral vectors from separate modules has been proven to be highly suitable for evaluating viral transgene expression in plants [17-19].

In the course of providing a plant-produced recombinant OspA vaccine we have recently shown that recombinant OspA can be produced in tobacco chloroplasts and that the protein is lipidated and immunogenic in mice [20]. However, while plants producing OspA at $1 \%$ of TSP were healthy, those accumulating OspA at 10\% TSP were hampered in photosynthesis and unable to grow photoautotrophically [15], limiting the suitability of this production system. To 
further understand and eventually overcome the effects which limit production of bacterial lipoproteins in chloroplasts, we wanted to use a proteomics approach to indentify potentially differences in the plant cell protein inventory. Moreover, we used a transient approach as an alternative to produce OspA variants in either lipidated or non-lipidated form to provide a plant made vaccine candidate against Lyme disease.

\section{MATERIALS AND METHODS}

\section{D-PAGE}

All chemicals and materials for 2D-PAGE were purchased from GE Healthcare, Munich, Germany. Sequencing grade modified trypsin was obtained from Promega, Mannheim, Germany. All other chemicals and HPLC-solvents were acquired from Merck KGaA, Darmstadt, Germany.

Total protein for 2D-PAGE was isolated from transplastomic plants grown under sterile conditions on sugar containing medium [15] after the method by [21]. Protein pellets were resuspended in R-buffer ( $7 \mathrm{M}$ urea, $2 \mathrm{M}$ thiourea, $2 \%$ CHAPS, $2 \%$ IPG buffer, $50 \mathrm{mM}$ DTT) and the protein content of each sample was determined using the 2D Quant assay (GE Healthcare, Munich, Germany). Equal amounts of proteins were separated by 2D-PAGE. Therefore, isoelectric focusing was performed using the Ettan ${ }^{\mathrm{TM}}$ IPGphor ${ }^{\mathrm{TM}}$ II IEF System (GE Healthcare) to a total of $52 \mathrm{kVh}$. IPG strips (24 $\mathrm{cm}, \mathrm{pH} 3-10$, non-linear) were incubated in $450 \mu \mathrm{L}$ reswelling buffer over night ( $7 \mathrm{M}$ urea, $2 \mathrm{M}$ thiourea, 2\% CHAPS, 2\% carrier ampholytes $\mathrm{pH} 3-10,50 \mathrm{mM}$ DTT). Of each protein $200 \mu \mathrm{g}$ (in $100 \mu \mathrm{l}$ ) were loaded on IPG strips using sample cups at the acidic end of the IPG strip. Before transferring IPG strips onto $12.5 \%$ SDS polyacrylamide gels, proteins were reduced using $65 \mathrm{mM}$ DTT and alkylated by $280 \mathrm{mM}$ iodoacetamide. Electrophoresis was performed using the EttanDalt ${ }^{\mathrm{TM}}$ system (GE Healthcare). Finally, gels were subjected to silver staining according to [22]. For image analysis, gels were scanned with standardized parameters and gel images were processed by the Delta2D ${ }^{\mathrm{TM}}$ Software (Decodon, Greifswald, Germany).

\section{LC-MS/MS-Analysis}

Sample preparation of all spots was performed according to the protocol of Shevchenko et al. [23] Briefly, gel spots were washed alternately twice for $10 \mathrm{~min}$ with $50 \mathrm{mM}$ $\mathrm{NH}_{4} \mathrm{HCO}_{3}$ and $25 \mathrm{mM} \mathrm{NH}_{4} \mathrm{HCO}_{3}$ in $50 \%$ acetonitrile. The protein spots were vacuum dried prior to tryptic digestion. Gel pieces were rehydrated with $12.5 \mathrm{ng} / \mu \mathrm{L}$ trypsin in $50 \mathrm{mM}$ $\mathrm{NH}_{4} \mathrm{HCO}_{3}$. Incubation was performed over night at $37^{\circ} \mathrm{C}$. The resulting peptides were extracted by addition of $15 \mu \mathrm{L}$ $5 \%$ formic acid for 10 minutes.

The extracted peptides were separated using Ultimate ${ }^{\mathrm{TM}}$ 3000 chromatography system (Dionex, Idstein, Germany) coupled to an ESI-MS. The sample was pre-concentrated on a $300 \mu \mathrm{m}$ ID $1 \mathrm{~mm} \mathrm{C}_{18}$ trapping column. Afterwards, peptides were separated on a $75 \mu \mathrm{m}$ ID $\mathrm{C}_{18} 15 \mathrm{~cm}$ main column by a binary gradient composed of solvent $\mathrm{A}(0.1 \%$ formic acid) and solvent B ( $0.1 \%$ formic acid in $84 \%$ acetonitrile). Solvent B was increased from $5 \%$ to $95 \%$ in 55 minutes. The flow rate was set to $250 \mathrm{~nL} / \mathrm{min}$. Peptides were directly eluted into the ion trap LCQ Deca XPplus ${ }^{\mathrm{TM}}$ (Thermo Elec- tron, Dreieich, Germany). MS-acquisition duty cycle was set up with $1 \mathrm{~s}$ survey scan and three dependent scans (each approximately $1 \mathrm{~s}$ ). [24]

Generated data was processed using the search algorithm MASCOT $^{\mathrm{TM}}$ (version 2.1, Matrix Science, London, UK [25]). Database searches were accomplished using SwissProt (version 2005, Geneva, Switzerland). As fixed modification carbamidomethylation of cysteines was selected and oxidation of methionine as optional modification. As further parameters tryptic digest and up to one missed cleavages were considered for the search algorithms. Singly, doubly and triply charged ions were considered, respective mass tolerances were set to $1.5 \mathrm{Da}$. For MASCOT ${ }^{\mathrm{TM}}$ the minimum score was set to 35 for each peptide. All spectra were verified manually in order to avoid false positive hits derived from the search algorithms. Furthermore, each protein identification was based on at least 2 validated MS/MS-spectra

\section{Vector Cloning}

For transient expression of OspA-variants, the sequence was PCR-amplified with primers adding a unique $B s a \mathrm{I}$ site five base pairs upstream of the coding sequence. Forward primers were 5'-AATTTGGTCTCAAGGTATGGCAAAAT ATTTATTGGGAATAGGTC-3' for OA25, 5'-TTTGGTCT CAAGGTATGAAAAAATATTTATTGGG-3' for OA27, 5'- TCTTTGGTCTCAAGGTCCTCCATGGGATGTAAGCAAAATGTTAGCAGCC-3' for OT22, and 5' -TTTGGTCT CAAGGTATGTGTAAGCAAAATG-3' for OT24. Reverse primer was as described in [15]. BsaI/XbaI-fragments were cloned into the magnICON 3 '-module cloning vector pICH10990 , resulting in vectors pOA6125, pOA6127, pOT6122, and pOT6124. Plasmids were subsequently transferred to Agrobacterium tumefaciens GV3101 as described in [26].

\section{Plant Transformation and Transient Expression}

Transient expression was achieved by infiltrating Agrobacterium suspension into leaves of Nicotiana benthamiana. Bacteria were grown to an OD600 of about 2, pelleted by centrifugation and resuspended in a five-fold volume of a solution containing $10 \mathrm{mM}$ MES (pH 5.5) and $10 \mathrm{mM}$ $\mathrm{MgSO}_{4}$. Suspensions of 3 '-modules containing the OspAsequences were mixed with equal volumes of bacteriasuspensions containing the $5^{\prime}$-module pICH15879 and the recombinase module pICH10881and infiltrated into leaves using a syringe without a needle. Ten days after infiltration leaves were harvested and frozen in liquid nitrogen prior to analysis.

\section{SDS-PAGE, Western Blotting, and Fluorography}

For detection of recombinant OspA in transformed plants, $100 \mathrm{mg}$ of frozen plant material was homogenized in $150 \mu \mathrm{L}$ $1 \times$ SDS-sample buffer. The samples were heated to $90^{\circ} \mathrm{C}$ for 10 minutes and applied to SDS-PAGE (12.5\%). For detection of YFP, frozen leaf tissue was homogenized and extracted with $1 \times$ PBS buffer. Protein content was determined with the BCA-protein assay kit (Pierce, Rockford, IL, USA) and $15 \mu \mathrm{g}$ protein were loaded onto SDS-PAGE (12.5\%).

Proteins were transferred to a PVDF membrane (Hybond$P$ PVDF-membrane, GE Healthcare) in a tank-blot-equipment at $100 \mathrm{~V}$ for one hour. After incubating with OspA- 
antibody (Fitzgerald, Concord, MA, USA) at 1:10,000 dilution, the membrane was washed for three times 15 minutes with $1 \times$ PBST buffer $\left(23 \mathrm{mM} \mathrm{Na} \mathrm{HPO}_{4}, 17 \mathrm{mM} \mathrm{Na}_{2} \mathrm{HPO}_{4}\right.$, $100 \mathrm{mM} \mathrm{NaCl}, 0.1 \%$ (v/v) Tween 20). The washing steps were repeated after incubating the membrane with the HRPconjugated secondary antibody (dilution 1:20,000) for one hour. The detection followed with SuperSignal West Pico Chemiluminescent Kit (Pierce) according to the manual of the supplier.

For isotope labelling, protoplasts were isolated as described by [27]. Approximately $5 \times 10^{5}$ protoplasts in $200 \mu \mathrm{l}$ solution were incubated with $200 \mu \mathrm{Ci}\left[9,10-{ }^{3} \mathrm{H}\right]$ palmitic acid in $8 \mu \mathrm{L}$ DMSO (25\%) for three hours. Isolation and detection of labelled proteins were done as described elsewhere [15].

For detection of OspA-synthesis in protoplasts, $20 \mu \mathrm{L}$ of isolated protoplasts were incubated with $5 \times \mathrm{SDS}$ sample buffer at $90^{\circ} \mathrm{C}$ for ten minutes. Western blot analysis of recombinant protein was done as described above.

\section{Quantification of Recombinant Protein}

To assess the amount of recombinant OspA accumulating after transient expression, crude extracts of total leaf protein was separated by SDS-PAGE. Along with the samples, defined amounts of pure recombinant bacterial OspA were separated. Proteins were transferred to a PVDF-membrane and detected as described above. Quantification was done in a ChemiDoc XRS imager (BioRad, Munich, Germany).

\section{RESULTS AND DISCUSSION}

\section{D-Gel-Electrophoresis}

Transplastomic tobacco plants accumulating recombinant OspA up to a level of $1 \%$ of the total soluble protein (TSP) were viable (line OA12) and almost indistinguishable from wild type (WT). In contrast, plants accumulating 10\% OspA (line OA13) were unable to perform photosynthesis and required sugar for growth [15].

To assess the differences between the two transplastomic lines and wild type we performed 2D-gel-electrophoresis of total cellular protein of the different plant lines. When comparing the gels only protein spots with an at least 3-fold change in abundance were considered differential. In lines OA12 and OA13, three additionally, highly abundant protein spots occurred (Fig. 1) which were identified as OspA using nano-LC-MS/MS (data not shown). In general, most of the spots in the 2D-gels of the transplastomic lines slightly decreased in comparison to WT, but less than factor 3. This might be due to the constant total protein amount applied to the gels, were high-level of recombinant protein is on the expense of total protein.

No significant increase in these spots was detected from the OA12- to the OA13-lines presumably due to the saturation of the silver stained spots limiting the dynamic range of the 2D-gel-electrophoresis. However, the phenotypical changes of the mutants compared to WT are not reflected in the 2D-gel-pattern representing mostly high-abundant, soluble proteins. Thus, these changes are either due to steric reasons or are induced by alteration of proteins not visible on the 2D-gels (e.g. low-abundant proteins).

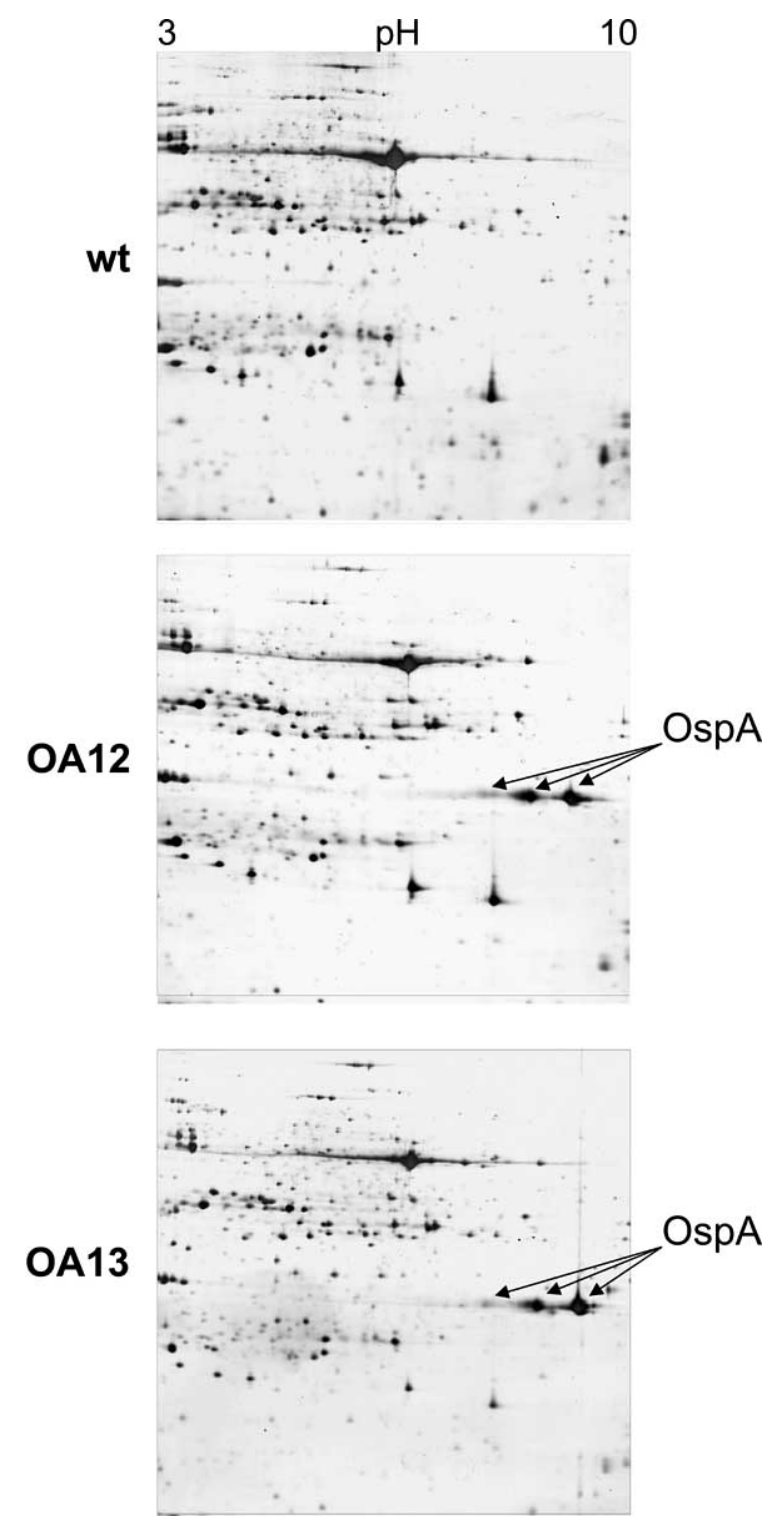

Fig. (1). 2D-PAGE of Nicotiana tabacum wild type (wt), OA12 and OA13 mutant leafs. While most of the protein spots are slightly down-regulated in the mutants three highly abundant protein spots arise from which OspA can be identified in all cases.

\section{Production of OspA Variants via a Transient Tobacco- Mosaic Virus-Based Expression System}

To evaluate OspA accumulation and palmitoylation in plant cells we used viral transient expression mediated by Agrobacterium transfection of Nicotiana benthamiana plants [17]. To determine protein palmitoylation in dependence of the signal sequence (SS), four different constructs were cloned (Table 1).

Variant OA27 exhibited the genuine OspA-sequence, while OA25 had an amino acid exchange in position 2 (Lys $\rightarrow$ Ala). The variant OT24 was lacking the N-terminal SS and was identical to the non-palmitoylated chloroplast variant OT11 [20]. Additionally, a truncated form OT22 was made which exhibited an N-terminal sequence (Met-GluCys-) known to lead to palmitoylation and/or myristoylation in eukaryotic cells (e.g. G-protein subunit $\alpha$ s [36]). This pro- 
tein should serve as a control to determine if a recombinant protein could be lipidated in the plant cell cytoplasm.

Table 1. N-terminal Sequences of Constructs Used in this Study

\begin{tabular}{|c|c|}
\hline Name of Construct & N-terminal Sequence \\
\hline \hline OA12/13 & MAKYLLGIGLILALIACKQ $\ldots$ \\
\hline OA25 & MAKYLLGIGLILALIACKQ ... \\
\hline OA27 & MKKYLLGIGLILALIACKQ ... \\
\hline OT22 & MGCKQ ... \\
\hline OT24 & \\
\hline
\end{tabular}

For Agrobacterium-mediated viral transient expression, plants were infiltrated with bacteria suspensions and crude leaf extracts were analyzed after ten days for OspAaccumulation.

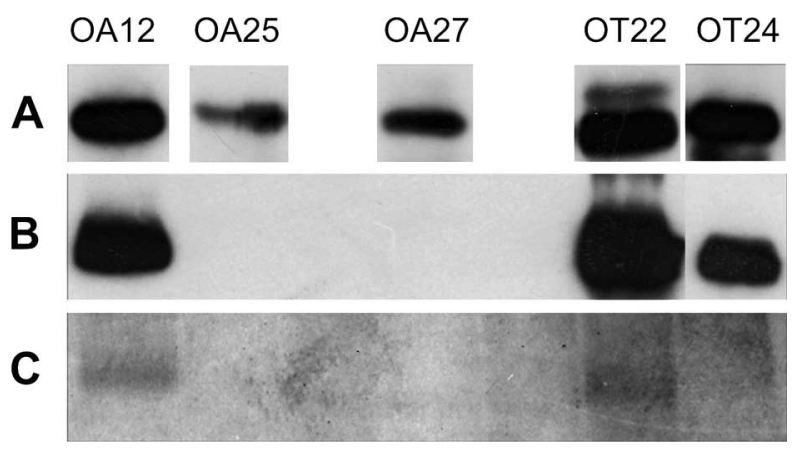

Fig. (2). Immunoblot and ${ }^{3} \mathrm{H}$-palmitic acid incorporation analysis of different OspA-variants after transient expression in Nicotiana benthamiana. (A) Crude extracts of total proteins after ten days from transfection were separated on SDS-PAGE and probed with anti-OspA monoclonal antibody. (B) Isolated protoplasts were lyzed, subjected to SDS-PAGE, and analyzed. (C) Protoplasts were incubated with ${ }^{3} \mathrm{H}$-palmitic acid and OspA was subsequently isolated via metal affinity chromatography. Protein was separated by SDS-PAGE and analyzed by fluorography. Note the slightly lower size of OT22 compared to OA12 due to the lack of the signal sequence in OT22.

As shown in Fig. (2A), all lines produced significant amounts of recombinant OspA (compared to OA12 from chloroplasts). To investigate protein palmitoylation, protoplasts were prepared from ten days old leaves and checked again for OspA-content (Fig. 2B). While OspA could be detected in lines OA12 (control), OT22, and OT24, no OspA was found in protoplasts of lines OA25 or OA27. Analysis of the bacterial SS of OspA with TargetP [29] provided evidence that the genuine OspA SS could act as a presequence for targeting the protein to the apoplast. In fact, a previous study showed that OspA is secreted when expressed in Nicotiana tabacum suspension cells [30]. Hence, full-length OspA variants could not be detected in protoplasts.

\section{Analysis of Palmitoylation}

To determine if OspA-variants are palmitoylated in the cytoplasm, labeling of isolated protoplasts with ${ }^{3} \mathrm{H}$-palmitic acid was performed. While the chloroplast-produced OA12 was palmitoylated (Fig. 2C), only OT22 from the cytosolproduced variants showed a label and OT24 did not. This is proof that the N-terminal Met-Gly-Cys-motif facilitates palmitoylation of the recombinant OspA in the cytosol while the motif Met-Cys- does not. However, eukaryotic SAcylation is clearly different from bacterial lipoproteins which exhibit a diacylglycerol attached to the thiol group of cysteine. It has been shown in the case of the Plasmodium falciparum antigen LSA that the addition of a single palmitic acid to a peptide strengthens its immunogenicity in the absence of adjuvant [31]. It needs to be tested in which way this modification modulates the immunogenicity of the recombinant OspA and if OT22 also could serve as a vaccine.

Since OA25 and OA27 are excluded from the protoplast, no palmitoylation could be detected. However, lipidation of full length OspA after transient expression is unlikely since prediction of the signal sequence indicated that the $\mathrm{N}$ terminal 23 amino acids are cleaved during transport to the apoplast. This would eliminate the sole cysteine in position 17 and therefore, palmitoylation of OspA could not occur.
OA25

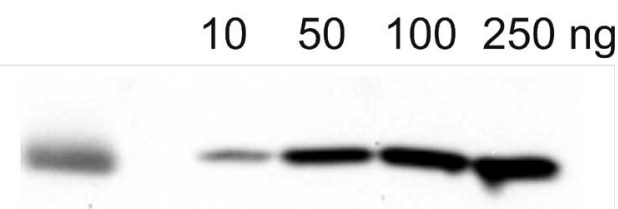

Fig. (3). Immunoblot for quantification of OspA after transient expression. Defined amounts of recombinant bacterial OspA (recO$\mathrm{spA}$ ) were applied to Western blot and intensities of signals compared to crude protein extracts from infiltrated plants (corresponding to $7 \mathrm{mg}$ of leaf). The amount of OspA from construct OA25 was determined as $15 \mu \mathrm{g} / \mathrm{g}$ fresh leaf.

Although no optimization of transient OspA-production was performed at this stage of the study, it was clearly shown that OspA could be produced at considerable levels compared to chloroplast produced OspA. For OA25 we determined an OspA amount of about $15 \mu \mathrm{g} / \mathrm{g}$ (Fig. 3), which is roughly $1 / 3$ of the OspA-content of the transplastomic line OA12. With the use of more advances expression vectors it should be feasible to further enhance this amount [32].

Especially the ease and speed of the system made rapid analysis of different constructs possible in a very short time (weeks) which should allow the identification of optimal conditions for expression.

\section{CONCLUSIONS}

Plants are an extremely versatile expression platform for recombinant therapeutic proteins. The use of different cellular compartments and/or expression systems (stable vs. transient) provides a toolbox to determine the optimal solution for each single protein. Moreover, different posttranslational modifications can be achieved by targeting proteins to subcellular locations and by utilizing either eukaryotic or prokaryotic signal sequences. As shown for OspA, several strategies should be examined to find optimal conditions for a given target protein. 


\section{ACKNOWLEDGEMENTS}

This work was supported by the Deutsche Forschungsgemeinschaft (DFG), grant WA1448/3 and GK1342 (Y.R.).

\section{REFERENCES}

[1] Sutcliffe IC, Russell RR. Lipoproteins of gram-positive bacteria. J Bacteriol 1995; 177: 1123-8.

[2] Hayashi S, Wu HC. Lipoproteins in bacteria. J Bioenerg Biomembr 1990; 22: 451-71.

[3] Rodriguez-Ortega MJ, Norais N, Bensi G, et al. Characterization and identification of vaccine candidate proteins through analysis of the group A Streptococcus surface proteome. Nat Biotechnol 2006; 24: 191-7.

[4] Masignani V, Comanducci M, Giuliani MM, et al. Vaccination against Neisseria meningitidis using three variants of the lipoprotein GNA1870. J Exp Med 2003; 197: 789-99.

[5] Sigal LH, Zahradnik JM, Lavin P, et al. A vaccine consisting of recombinant Borrelia burgdorferi outer-surface protein A to prevent Lyme disease. Recombinant Outer-Surface Protein A Lyme Disease Vaccine Study Consortium. N Engl J Med 1998; 339: 21622 .

[6] Steere AC, Sikand VK, Meurice F, et al. Vaccination against Lyme disease with recombinant Borrelia burgdorferi outer-surface lipoprotein A with adjuvant. Lyme Disease Vaccine Study Group. N Engl J Med 1998; 339: 209-15.

[7] Erdile LF, Brandt MA, Warakomski DJ, et al. Role of attached lipid in immunogenicity of Borrelia burgdorferi OspA. Infect Immun 1993; 61: 81-90.

[8] Weis JJ, Ma Y, Erdile LF. Biological activities of native and recombinant Borrelia burgdorferi outer surface protein A: dependence on lipid modification. Infect Immun 1994; 62: 4632-6.

[9] Taylor PD, Toseland CP, Attwood TK, et al. LIPPRED: A web server for accurate prediction of lipoprotein signal sequences and cleavage sites. Bioinformation 2006; 1: 176-9.

[10] Dunn JJ, Lade BN, Barbour AG. Outer surface protein A (OspA) from the Lyme disease spirochete, Borrelia burgdorferi: high level expression and purification of a soluble recombinant form of OspA. Protein Expr Purif 1990; 1: 159-68.

[11] Warzecha H, Mason HS. Benefits and risks of antibody and vaccine production in transgenic plants. J Plant Physiol 2003; 160: 755-64.

[12] Streatfield SJ. Approaches to achieve high-level heterologous protein production in plants. Plant Biotechnol J 2007; 5: 2-15.

[13] Tregoning JS, Nixon P, Kuroda $\mathrm{H}$, et al. Expression of tetanus toxin Fragment $\mathrm{C}$ in tobacco chloroplasts. Nucleic Acids Res 2003; 31: 1174-9.

[14] Molina A, Hervas-Stubbs S, Daniell H, et al. High-yield expression of a viral peptide animal vaccine in transgenic tobacco chloroplasts. Plant Biotechnol J 2004; 2: 141-53.

[15] Hennig A, Bonfig K, Roitsch T, et al. Expression of the recombinant bacterial outer surface protein A in tobacco chloroplasts leads to thylakoid localization and loss of photosynthesis. FEBS J 2007; 274: 5749-58.
[16] Pogue GP, Lindbo JA, Garger SJ, et al. Making an ally from an enemy: plant virology and the new agriculture. Annu Rev Phytopathol 2002; 40: 45-74

[17] Marillonnet S, Giritch A, Gils M, et al. In planta engineering of viral RNA replicons: efficient assembly by recombination of DNA modules delivered by Agrobacterium. Proc Natl Acad Sci U S A 2004; 101: 6852-7.

[18] Marillonnet S, Thoeringer C, Kandzia R, et al. Systemic Agrobacterium tumefaciens-mediated transfection of viral replicons for efficient transient expression in plants. Nat Biotechnol 2005; 23: 71823.

[19] Gils M, Kandzia R, Marillonnet S, et al. High-yield production of authentic human growth hormone using a plant virus-based expression system. Plant Biotechnol J 2005; 3: 613-20.

[20] Glenz K, Bouchon B, Stehle T, et al. Production of a recombinant bacterial lipoprotein in higher plant chloroplasts. Nat Biotechnol 2006; $24: 76-7$

[21] Wang W, Vignani R, Scali M, et al. A universal and rapid protocol for protein extraction from recalcitrant plant tissues for proteomic analysis. Electrophoresis 2006; 27: 2782-6.

[22] Blum H, Beier H, Gross HJ. Improved silver staining of proteins, RNA, DNA in polyacrylamide gels. Electrophoresis 1987; 8: 93-9.

[23] Shevchenko A, Wilm M, Vorm O, et al. Mass spectrometric sequencing of proteins silver-stained polyacrylamide gels. Anal Chem 1996; 68: 850-8.

[24] Mitulovic G, Smoluch M, Chervet JP, et al. An improved method for tracking and reducing the void volume in nano HPLC-MS with micro trapping columns. Anal Bioanal Chem 2003; 376: 946-51.

[25] Hirosawa M, Hoshida M, Ishikawa M, et al. MASCOT: multiple alignment system for protein sequences based on three-way dynamic programming. Comput Appl Biosci 1993; 9: 161-7.

[26] Chen H, Nelson RS, Sherwood JL. Enhanced recovery of transformants of Agrobacterium tumefaciens after freeze-thaw transformation and drug selection. Biotechniques 1994; 16: 664-8.

[27] Treuter E, Nover L, Ohme K, et al. Promoter specificity and deletion analysis of three heat stress transcription factors of tomato. Mol Gen Genet 1993; 240: 113-25.

[28] Smotrys JE, Linder ME. Palmitoylation of intracellular signaling proteins: regulation and function. Annu Rev Biochem 2004; 73: 559-87.

[29] Emanuelsson O, Brunak S, von Heijne G, et al. Locating proteins in the cell using TargetP, SignalP and related tools. Nat Protoc 2007; 2: 953-71.

[30] Navarre C, Delannoy M, Lefebvre B, et al. Expression and secretion of recombinant outer-surface protein A from the Lyme disease agent, Borrelia burgdorferi, in Nicotiana tabacum suspension cells. Transgenic Res 2006; 15: 325-35.

[31] BenMohamed L, Belkaid Y, Loing E, et al. Systemic immune responses induced by mucosal administration of lipopeptides without adjuvant. Eur J Immunol 2002; 32: 2274-81.

[32] Giritch A, Marillonnett S, Engler C, et al. Rapid high-yield expression of full-size IgG antibodies in plants coinfected with noncompeting viral vectors. Proc Natl Acad Sci USA 2006; 103: 17678-83. 